\title{
Septicaemia caused by cysteine-requiring isolates of Escherichia coli
}

\author{
J. W. TAPSALL and C. J. MCIVER
}

Department of Microbiology, The Prince of Wales Hospital, Randwick, Sydney, New South Wales, Australia 2031

\begin{abstract}
Summary. The clinical and bacteriological findings in five cases of septicaemia with cysteine-requiring isolates of Escherichia coli are reported. Infections with these nutritionally-dependent organisms have been found previously in the urinary tract only, associated usually with chronic rather than acute conditions. The urinary tract was considered to be the source of the septicaemia in our patients and that site should be investigated when such strains are isolated from blood cultures. When first isolated the organisms characteristically form small translucent colonies on media deficient in appropriate growth factors. Their nutritional requirement for cysteine can be determined by a simple auxanographic technique, thereby enabling the appropriate supplementation of media necessary for reliable identification and antibiotic-sensitivity testing.
\end{abstract}

\section{Introduction}

Bacteria that require a specific nutrient for optimal growth are not infrequently isolated from clinical material. Strains of nutritionally-dependent Enterobacteriaceae typically form small translucent colonies on media deficient in the required growth factor(s) and are referred to as "smallcolony forms" (Gillespie, 1952) or "deficient dwarf colonies" (Borderon and Horodniceanu, 1978). The nutritional defects most commonly reported are in the metabolism of sulphur, the so-called "cysteine-requiring strains" (Gillespie, 1952), or in thymidine synthesis (Tapsall et al., 1974; Borderon and Horodniceanu, 1978).

Urinary isolates of Escherichia coli that were cysteine-requiring were described by Gillespie (1952) and bacteriological media specially formulated to facilitate the isolation of these organisms have subsequently come into widespread use (Mackey and Sandys, 1966; Bevis, 1968). Despite awareness of these strains and the use of media suitable for their growth, naturally occurring cysteine-requiring isolates of $E$. coli have hitherto been isolated only from the urinary tract. We report here five cases of septicaemia due to cysteinerequiring isolates of $E$. coli. Potential difficulties relating to the isolation, identification and sensitivity testing of these strains are discussed.

Received 6 Mar. 1986; accepted 1 Apr. 1986.

\section{Materials and methods}

\section{Patients}

The clinical features of the five patients with septicaemia caused by cysteine-requiring isolates of $E$. coli are shown in table I.

\section{Bacteriological methods}

Blood for culture was inoculated in 5-ml volumes into $45 \mathrm{ml}$ each of Tryptone Soya Broth (Oxoid) and Nutrient Broth No. 2 (Oxoid) to which additions of various nutrients, including L-cysteine hydrochloride (British Drug Houses) at a concentration of $2.5 \mathrm{mmol}$, had been made. Routine subcultures were made on to layered nutrient horse-blood agar after incubation for 1,2 and 7 days. The filter-paper technique of Leigh and Williams (1964) and MacConkey Agar (Oxoid) were used to quantitate bacterial growth from urine samples as previously described (Tapsall et al., 1975).

Isolates were identified by the methods of Cowan (1974) and by the API 20E kit (API Systems) with inocula derived from isolation media. These procedures were repeated with inocula supplemented with $0.63 \mathrm{~mm}$ L-cysteine hydrochloride.

Sensitivity tests were performed according to the calibrated dichotomous sensitivity (CDS) method (Bell, 1975) with Sensitest Agar (Oxoid). Sensitivity tests were also performed on Isosensitest Agar (Oxoid) and Mueller-Hinton Agar (Difco). The inoculum for these tests was prepared in physiological saline and also in physiological saline with L-cysteine hydrochloride added to a concentration of $0.63 \mathrm{mmol}$. Requirements for cysteine were determined by a variation of the auxano- 
Table I. Clinical presentations of five patients with septicaemia caused by cysteine-requiring $E$. coli

\begin{tabular}{|c|c|c|c|c|c|c|}
\hline Patient & Age & Sex & Presentation & Site & Predisposing condition & Outcome \\
\hline 1 & 80 & $\mathrm{~F}$ & Acute cardiac failure & Blood & IDC & $\begin{array}{l}\text { Cardiac death and acute suppura- } \\
\text { tive pyelonephritis at autopsy }\end{array}$ \\
\hline 2 & 60 & $\mathrm{~F}$ & Acute septicaemia & $\begin{array}{l}\text { Blood } \\
\text { Urine }\end{array}$ & Multiple myeloma & Recovered with antibiotic therapy \\
\hline 3 & 59 & $\mathrm{~F}$ & Acute cardiac failure & Blood & IDC; diabetes & $\begin{array}{l}\text { Hyperosmolar diabetic coma and } \\
\text { cardiac death }\end{array}$ \\
\hline 4 & 53 & $\mathrm{~F}$ & $\begin{array}{l}\text { Recurrent septicaemia; } \\
3 \text { episodes over } 3 \text { months }\end{array}$ & $\begin{array}{l}\text { Blood } \\
\text { Urine } \\
\text { Renal abscess } \\
\text { Wound abscess }\end{array}$ & $\begin{array}{l}\text { Renal transplant; } \\
\text { immunosuppressed }\end{array}$ & $\begin{array}{l}\text { Ultimately recovered with anti- } \\
\text { biotics and surgical drainage }\end{array}$ \\
\hline 5 & 57 & $\mathrm{~F}$ & $\begin{array}{l}\text { Acute pelvi-calyceal urinary } \\
\text { obstruction }\end{array}$ & $\begin{array}{l}\text { Urine } \\
\text { Blood }\end{array}$ & $\begin{array}{l}\text { Diabetes; papillary } \\
\text { necrosis }\end{array}$ & $\begin{array}{l}\text { Recovered with antibiotics and } \\
\text { surgical drainage }\end{array}$ \\
\hline
\end{tabular}

$\mathrm{IDC}=$ indwelling urinary catheter.

graphic technique for demonstrating thymidine dependence (Tapsall et al., 1974). The surface of a plate of Sensitest agar was flooded with a suspension of the organism as for CDS-sensitivity testing. Paper disks impregnated with a $0.63 \mathrm{~mm}$ solution of L-cysteine hydrochloride were placed on the surface. Growth enhancement around the disk indicated a requirement for cysteine.

The isolates were phenotyped according to the classification of Jones-Mortimer (1968) on Davis and Mingioli basal medium as modified by Pasternak (1962). Briefly. the organisms were grown overnight in modified Davis and Mingioli broth with added L-cysteine hydrochloride $(0.63 \mathrm{~mm})$; thereafter they were centrifuged and washed three times with. and resuspended in. physiological saline to an optical density of 0.75 at $640 \mathrm{~nm}$ as determined on a Bausch and Lomb Spectronic 20 spectrophotometer. A $0.025-\mathrm{ml}$ volume of this suspension was delivered on to a series of agar plates by means of a Steers-type replicating device. The series of plates comprised the basal medium alone or supplemented with: $10 \mathrm{~mm} \mathrm{Na} \mathrm{SO}_{4}$ (Merck): $0.5 \mathrm{~mm}$ cysteine sulphinic acid (Sigma) (Dreyfuss and Monty. 1963): $0.42 \mathrm{mM} \mathrm{Na} \mathrm{Na}_{2}$ (Sigma): or $0.85 \mathrm{mM}$ L-cysteine (Sigma). The inoculated plates were incubated overnight at $37 \mathrm{C}$ and examined for growth after incubation for 24,48 and $72 \mathrm{~h}$. E. coli strain NCTC 10418 served as a control in these tests.

\section{Results}

The small-colony isolates from these five patients required cysteine for satisfactory growth on isolation and Sensitest agars. Without adequate cysteine supplementation, they formed the small translucent colonies (c. $0.5 \mathrm{~mm}$ diameter) typical of these strains. In the presence of $0.63 \mathrm{~mm}$ cysteine hydrochloride, colony diameter increased to $2 \mathrm{~mm}$ but the translucent appearance was retained. The requirement for cysteine was confirmed by the auxanographic technique when growth or enhanced growth occurred around the cysteineimpregnated disk (fig).

Isolates from patients 1-5 grew on basal medium supplemented with cysteine sulphinic acid, cysteine or sodium sulphide but not with sodium sulphate. Thus, these isolates had a defect in the ability to reduce sulphate to sulphite and belonged phenotypically to the nutritional class ACDH of JonesMortimer (1968). (The nutritional class ACDH comprises strains with mutant loci which affect sulphate permease, other enzymes involved in the reduction of sulphate, or both).

The organisms were identified as $E$. coli by conventional tests and by the API $20 \mathrm{E}$ system. With the API 20E system a 48-h incubation period was required for identification, even with cysteine supplementation. After incubation for $24 \mathrm{~h}$ some reactions, especially those with the carbohydrateutilisation tests, were delayed and could not be interpreted in this system. Conventional tests too, especially those for lysine decarboxylase and urea hydrolysis (both made with Oxoid media), could not be interpreted due to lack of growth in the $a b$ sence of cysteine supplements.

All isolates grew poorly on Sensitest agar without added cysteine (fig.) but grew adequately on Isosensitest and Mueller-Hinton agars. Isosensitest agar contains cysteine $\mathrm{HCl}$ at a concentration of $0.13 \mathrm{mmol}$.

The isolates from these five patients, as well as having slightly different API-profiles, had different antibiotic-sensitivity patterns (table II). Nine isolates available from the blood, urine and abscesses of patient 4 had identical API profiles, gave identical reactions in conventional test systems and had the same sensitivity patterns to antibiotics tested. 


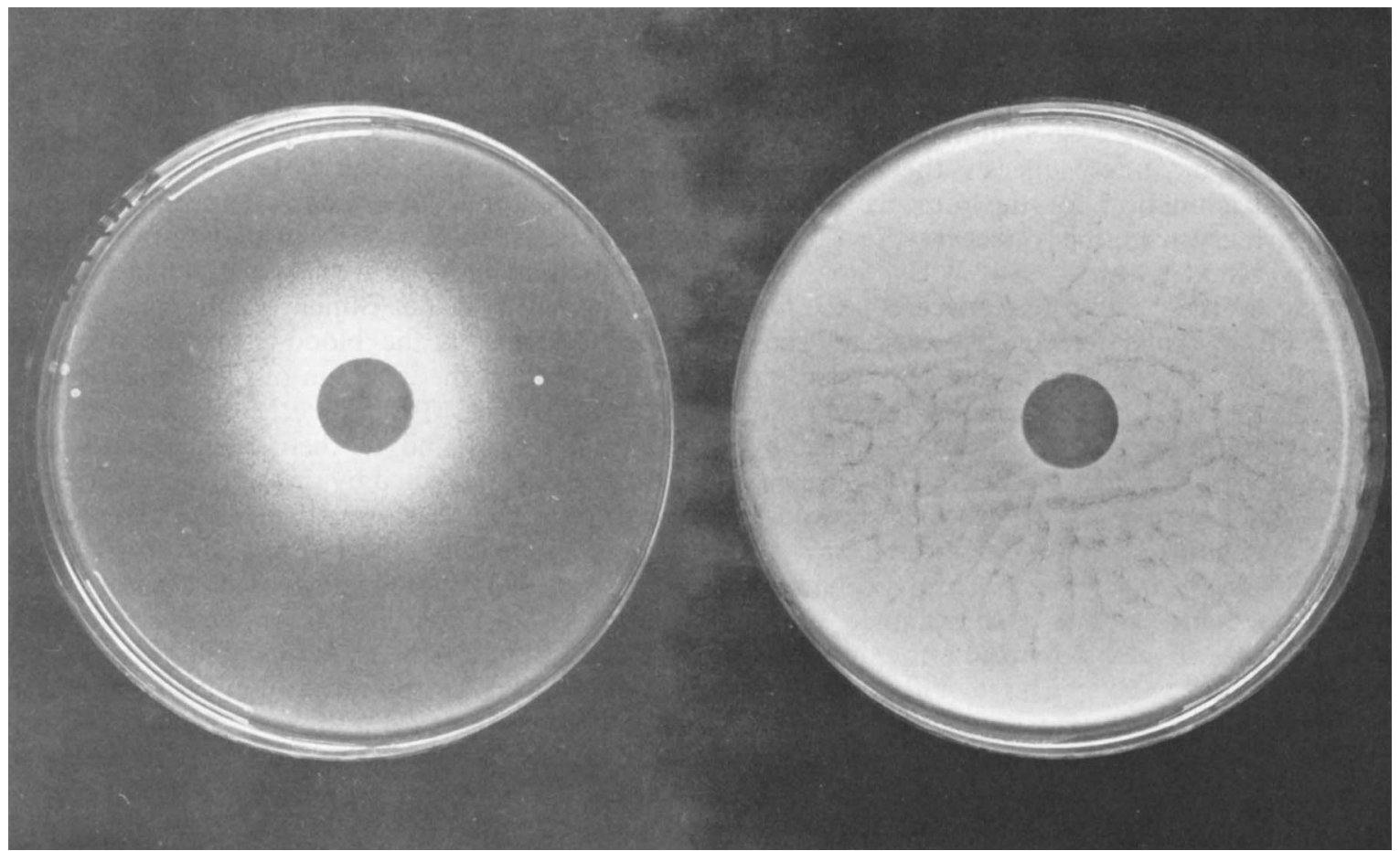

Fig. Growth of cysteine-requiring (left) and prototrophic control (right) strains of $E$. coli on Sensitest agar with disks impregnated with L-cysteine hydrochloride ( $0.63 \mathrm{~mm})$.

Table II. Responses of cysteine-requiring isolates of $E$. coli to various antibiotics

\begin{tabular}{lllllllll}
\hline $\begin{array}{l}\text { Isolate } \\
\text { from } \\
\text { patient }\end{array}$ & Sul & Tri & Amp & Gent & Chl & Tet & NA & Fur \\
\cline { 2 - 8 } & R & S & R & S & S & R & S & S \\
2 & R & R & R & S & S & S & R & S \\
3 & R & R & S & S & S & S & R & S \\
4 & R & S & R & S & R & R & S & S \\
5 & R & S & S & S & S & S & S & S \\
\hline
\end{tabular}

$\mathrm{Sul}=$ sulphonamide; Tri = trimethoprim; Amp = ampicillin; Gent = gentamicin; $\mathrm{Chl}=$ chloramphenicol; Tet = tetracycline; NA = nalidixic acid; Fur $=$ nitrofurantoin. For concentrations of antibiotics used, see Bell (1975).

$\mathrm{R}=$ resistant $\mathrm{S}=$ sensitive.

\section{Discussion}

Cysteine-requiring strains of $E$. coli have not been isolated previously from blood cultures or wound abscesses, earlier reports of infections with these strains referring only to the urinary tract (Gillespie, 1952; Borderon and Horodniceanu 1978). It is most likely that the urinary tract was the source of the septicaemia in these five patients. In three cases, the organism was isolated from the urine as well as from the blood and with the other two patients septicaemia followed the use of an indwelling urinary catheter.

The chronic nature of the urinary-tract infection was also emphasised in these earlier reports. However, the clinical presentation of the patients studied here suggested that these strains have the capacity to cause acute infections as well as chronic urinary-tract colonisation. Although the possibility of a chronic urinary-tract involvement could not be excluded, the findings at necropsy for patient 1 revealed only an acute suppurative pyelonephritis 
without evidence of chronic renal disease; the presentations of patients 2 and 3 were also consistent with an acute, rather than an acute-on-chronic infection. The strong association of these strains with the urinary tract suggests the need for a thorough examination for the presence in a coexisting urinary infection whenever they are isolated from blood cultures.

Amongst the Enterobacteriaceae, a large number of metabolic defects giving rise to "smallcolony forms" has been reported (Borderon and Horodniceanu, 1978). The general nature of the defect in the isolates studied here was determined by a simple auxanographic method with cysteineimpregnated paper disks. The specific disorder of sulphur metabolism was not determined but it was shown that these organisms lacked the capacity to reduce sulphate to sulphite. Their enhanced growth in the presence of added cysteine was explained by the ability of these organisms to utilise other sulphur-containing compounds thereby bypassing the metabolic defect. The precise requirements of these organisms for cysteine have not been determined in vitro but it is likely that growth in vivo is also enhanced in the presence of cysteine. Urinary excretion of cystine in amounts of $16.5-33.0 \mu \mathrm{mol}$ (2 $4 \mathrm{mg} \mathrm{L}$ ) and of the oxidation product, cysteine.

\section{REFERENCES}

Bell S M 1975 The CDS disc method of antibiotic sensitivity testing (calibrated dichotomous sensitivity test). Pathology 7 Suppl: 148

Bevis T D 1968 A modified electrolyte deficient culture medium. Journal of Medical Lahoratory Technology 25:38 41.

Borderon E. Horodniceanu T 1978 Metabolically deficient dwarf-colony mutants of Escherichia coli: deficiency and resistance to antibiotics of strains isolated from urine culture. Journal of Clinical Microbiology 8:629 634

Brigham M P. Stein W H. Moore S 1960 The concentrations of cysteine and cystine in human blood plasma. Journal of Clinical Investigation 39:1633 1638

Cowan S T 1974 Cowan and Steel's Manual for the identification of medical bacteria, 2nd edn. Cambridge University Press, Cambridge, pp 103-107.

Dreyfuss J. Monty K J 1963 The biochemical characterization of cysteine-requiring mutants of Salmonella Iyphimurium. Journal of Biological Chemistry 238:1019 1024 in concentrations of $41 \cdot 6-62 \cdot 4 \mu \mathrm{mol}(10-15 \mathrm{mg} / \mathrm{L})$ have been reported in fasting patients; again, levels of $41.6 \mu \mathrm{mol}(10 \mathrm{mg} / \mathrm{L})$ of cystine and $33.0 \mu \mathrm{mol}$ $(4 \mathrm{mg} / \mathrm{L})$ of cysteine have been found in the plasma of adults (Brigham et al., 1960).

The isolation of cysteine-requiring "smallcolony forms" of $E$. coli in clinical laboratories has been facilitated by use of bacteriological media with added cysteine. Similarly, the presence of added cysteine in the blood-culture media used here assisted in the isolation of these nutritionally aberrant strains. Tests for the identification of these organisms and of their sensitivity to antibiotics are also affected by this nutritional defect if these tests are performed on unsupplemented media. A previous report (Borderon and Horodniceanu, 1978) noted decreased sensitivity of "deficient dwarf colonies" to aminoglycoside antibiotics. The small-colony forms of $E$. coli reported here did not differ significantly in their antibioticsensitivity patterns from those of other $E$. coli isolates in this laboratory and all were sensitive to gentamicin in vitro. Furthermore, the outcome of therapy corresponded well with the sensitivity predicted by in-vitro testing, suggesting that these strains were not refractory to antibiotic treatment in the absence of complicating factors.

Gillespie W A 1952 Biochemical mutants of coliform bacilli in infections of the urinary tract. Journal of Pathology and Bacteriology 64:551 557.

Jones-Mortimer M C 1968 Positive control of sulphate reduction in Escherichia coli. Isolation. characterization and mapping of cysteineless mutants of $E$. coli K12. Biochemical Journal 110:589 595.

Leigh D A, Williams J D 1964 Method for the detection of significant bacteriuria in large groups of patients. Journal of Clinical Pathology 17:498 503.

Mackey J P. Sandys G H 1966 Diagnosis of urinary infections. British Medical Journal 1:1173.

Pasternak C A 1962 Sulphate activation and its control in Escherichia coli and Bacillus suhtilis. Biochemical Journal 85: 44 49.

Tapsall J W, Wilson E, Harper J 1974 Thymine dependent strains of Escherichia coli selected by trimethoprimsulphamethoxazole therapy. Pathology 6:161-167.

Tapsall J W. Taylor P C. Bell S M, Smith D D 1975 Relevance of "significant bacteriuria" to aetiology and diagnosis of urinary tract infection. Lancet 2:637-639. 